\title{
JVN observations of NLS1 galaxies and BAL quasars
}

\author{
Akihiro Doi*i; ${ }^{i a}$ Keiichi Asada, ${ }^{a}$ Hiroshi Nagai, ${ }^{b}$ Kenta Fujisawa, ${ }^{c}$ and Noriyuki \\ Kawaguchi ${ }^{b}$ \\ ${ }^{a}$ Institute of Space and Astronautical Science, Japan Aerospace Exploration Agency \\ 3-1-1 Yoshinodai, Sagamihara, Kanagawa 229-8510, Japan \\ ${ }^{b}$ National Astronomical Observatory of Japan \\ 2-21-1 Osawa, Mitaka, Tokyo 181-8588, Japan \\ ${ }^{c}$ Faculty of Science, Yamaguchi University \\ 1677-1 Yoshida, Yamaguchi, Yamaguchi 753-8512, Japan
}

We are conducting VLBI observations for Narrow-Line Seyfert 1 galaxies (NLS1s) and Broad Absorption Line (BAL) quasars, which are thought to be driven by highly accreting central engines, in order to understand accretion phenomena on such central engines. Because these AGN subclasses are generally very weak radio sources, phase-referencing VLBI observations are being carried out using Japanese VLBI Network (JVN), together with VLBA, EVN, and an opticalfibre-linked JVN subarray "OCTAVE." For NLS1s, 12 out of 14 sources have been successfully imaged in milli-arcsecond resolutions. For BAL quasars, 21 out of 23 sources have been successfully detected with OCTAVE baselines at $8.4 \mathrm{GHz}$. Several NLS1s and BAL quasars are investigated in images at multi-frequency. Some evidence of Doppler-boosting on relativistic jets were seen in several radio-loud objects, which is inconsistent with the edge-on view paradigm of BAL quasars.

The 9th European VLBI Network Symposium on The role of VLBI in the Golden Age for Radio Astronomy and EVN Users Meeting

September 23-26, 2008

Bologna, Italy

\footnotetext{
*Speaker.

${ }^{\dagger}$ E-mail: akihiro.doi@vsop.isas.jaxa.jp
} 


\section{Introduction}

Narrow-Line Seyfert 1 galaxies (NLS1s) and Broad Absorption Line (BAL) quasars are thought to be driven by accretion disks with high accretion rates onto super-massive black holes at the centers of galaxies. Accretion phenomena in these active galactic nuclei (AGNs) have not been well-understood. Very-Long-Baseline Interferometers (VLBIs) in radio bands offer an exclusive chance to reveal the parsec-scale structures in AGNs by direct imaging. Both of NLS1s and BAL quasars are relatively radio-weak AGNs. We have started to study these weak sources with VLBIs.

\section{Observations and Results}

\subsection{Narrow-Line Seyfert 1 galaxies}

We have observed both of radio-loud and radio-quiet NLS1s. The most radio-loud NLS1, SDSS J094857.3+002225, showed very compact structures and an inverted radio spectrum in our VLBA images at 1.7, 2.3, 5, 8.4, and $15 \mathrm{GHz}$, suggesting Doppler-boosting with a Doppler factor $\delta>2.7-5.5$ [1]. Japanese VLBI Network (JVN; [2]) observations at $8.4 \mathrm{GHz}$ and VLBA observations at $1.7 \mathrm{GHz}$ have revealed that other five radio-loud NLS1s included both steep-spectrum sources with extended structures, and inverted-spectrum sources with compact structures [3]. Six out of eight nearby radio-quiet NLS1s have been detected using the VLBA at $1.7 \mathrm{GHz}$. It has been found that a large fraction of radio emission originates in diffuse components resolved out, although these NLS1s are compact in arcsec resolutions. These results suggested that NLS1s are also an AGN class that can be detected with VLBIs, implying the existence of synchrotron component, presumably nonthermal jet. Although NLS1s are thought to have relatively small mass black holes, some of radio-loud NLS1s have the ability of generating relativistic jets.

\subsection{Broad Absorption Line quasars}

We have observed many radio-loud BAL quasars at redshift $z \sim 2$ selected from the Sloan Digital Sky Survey (SDSS) Third Data Release. We detected 21 out of 23 objects using an opticalfiber-linked real-time VLBI instrument constructed by the Optically ConnecTed Array for VLBI Exploration project (OCTAVE; [4]), which is operated as a subarray of the JVN. The OCTAVE connects six radio telescopes, Usuda $64 \mathrm{~m}$, Nobeyama $45 \mathrm{~m}$, Kashima $34 \mathrm{~m}$, Yamaguchi $32 \mathrm{~m}$, Tsukuba $32 \mathrm{~m}$, and Gifu $11 \mathrm{~m}$, using optical fibers at $2.4 \mathrm{Gbps}$, and offers mJy-level fringe detection sensitivities. The high detection rate in the VLBI baselines indicates that radio-loud BAL quasars have nonthermal jets in parsec scales. We suggest that the accretion disks of these radio-loud BAL quasars can generate radiation-pressure driven thermal outflows and magnetic-driven nonthermal jets simultaneously. We found four BAL quasars showing inverted spectra in our sample. Two of them have been imaged using JVN at 2.3, 6.7, and 8.4 GHz, and European VLBI Network (EVN) at $1.7 \mathrm{GHz}$. Both sources showed compact morphology and peaked spectra, which were apparently consistent with the properties of Compact Steep Spectrum (CSS) sources and Gigahertz-Peaked Spectrum (GPS) sources (cf. [5]). In addition to previously VLBI-detected BAL quasars [6-8], our OCTAVE observations have increased the number of VLBI-detected BAL quasars to about 40. 


\section{References}

[1] A. Doi et al., VLBI Observations of the Most Radio-Loud, Narrow-Line Quasar SDSS J094857.3+002225, PASJ 58829

[2] K. Fujisawa, Recent Results of the Japanese VLBI Network, in proceedings of the 9th European VLBI Network Symposium, PoS (IX EVN Symposium) 075

[3] A. Doi et al., Japanese VLBI Network Observations of Radio-Loud Narrow-Line Seyfert 1 Galaxies, PASJ 59703

[4] N. Kawaguchi, eVLBI Technical Development in Japan, in proceedings of the 9th European VLBI Network Symposium, PoS (IX EVN Symposium) 041

[5] F. M. Montenegro-Montes, K.-H. Mack, M. Vigotti, C. R. Benn, R. Carballo, J. I. González-Serrano, J. Holt, \& F. Jiménez-Luján, Radio spectra and polarization properties of radio-loud broad absorption-line quasars, MNRAS 3881853

[6] Jiang, D. R., \& Wang, T. G., EVN observation of three Broad Absorption Line quasars, A\&A, 397 L13

[7] M. Kunert-Bajraszewska, High-resolution imaging of compact HiBAL quasar 1045+352, in proceedings of the 9th European VLBI Network Symposium, POS (IX EVN Symposium) 018

[8] F.M. Montenegro-Montes, VLBA imaging of radio-loud BAL QSOs, in proceedings of the 9th European VLBI Network Symposium, PoS (IX EVN Symposium) 019. 